\title{
Focused ion beam induced synthesis of a porous antimony nanowire network
}

\author{
Christoph Schoendorfer, ${ }^{\text {a) }}$ Alois Lugstein, Youn-Joo Hyun, and Emmerich Bertagnolli \\ Institute for Solid State Electronics, Vienna University of Technology, Floragasse 7, A-1040 \\ Vienna, Austria \\ Lothar Bischoff \\ Institute of Ion Beam Physics and Materials Research, Research Center Dresden-Rossendorf Inc., POB \\ 510119, D-01314 Dresden, Germany \\ Philipp M. Nellen and Victor Callegari \\ EMPA, Swiss Federal Laboratories for Materials Testing and Research, Überlandstrasse 129, CH-8600 \\ Dübendorf, Switzerland \\ Peter Pongratz \\ Institute for Solid State Physics, Vienna University of Technology, Wiedner Hauptstraße 8-10, A-1040 \\ Vienna, Austria
}

(Received 22 February 2007; accepted 2 July 2007; published online 24 August 2007)

\begin{abstract}
We present a focused ion beam-based approach for the synthesis of an antimony nanofiber network. The nanofibers, with a homogeneous distribution of diameters of about $25 \mathrm{~nm}$ and lengths up to several microns, are synthesized in a self-assembling process without any additional material source at room temperature. It is possible to recrystallize the as-grown amorphous nanofibers by moderate rapid thermal annealing at $473 \mathrm{~K}$. These results have been verified by means of scanning electron microscopy, Auger electron spectroscopy, high-resolution transmission electron microscopy, selected area electron diffraction, and energy dispersive x-ray analysis. As this approach is not limited solely to the material discussed here, other substrates (e.g., GaSb and $\mathrm{Ge}$ ) and ion sources should extend this method to other materials, which offers a great potential for future nanoscale devices and applications. (C) 2007 American Institute of Physics. [DOI: 10.1063/1.2771044]
\end{abstract}

\section{INTRODUCTION}

By decreasing the critical dimensions of electronic devices down to the nanometer regime, the onset of quantum size effects may open a route to alternative device concepts. To gain full use of these envisaged quantum size effects, structures with sizes comparable to, or even less than, the de Broglie wavelength are required. For antimony $(\mathrm{Sb})$, which is the focus of our research, this is the case for structures smaller than $40 \mathrm{~nm} .{ }^{1,2}$ As theoretically shown, a further decrease of the bulk material to $2 \mathrm{D}$ films with thicknesses below $10 \mathrm{~nm}$ finally should lead to a transition from a semimetal to semiconducting $\mathrm{Sb}$, resulting in a significant change of the electron transport properties. ${ }^{3}$

Low-dimensional nanostructures are usually fabricated using either a top-down or a bottom-up strategy. The former technique is extremely flexible, but suffers from limitations in minimum feature size and uniformity. The latter one, utilizing spontaneous self-ordering effects, is limited by the broad size distribution and the lack of control of the positioning of the self-organized nanostructures. In this context the discovery of the appearance of periodic structures with dimensions in the nanometer regime induced by ion bombardment has attracted growing interest due to the possibility of obtaining a self-organized formation of nanometer structures. $^{4-7}$

\footnotetext{
${ }^{a)}$ Electronic mail: christoph.schoendorfer@tuwien.ac.at
}

Appleton et al. found that heavy-ion implantation leads to the formation of craters with diameters of about $20 \mathrm{~nm}$ due to morphological instabilities in the amorphous phase of initial crystalline Ge. ${ }^{8}$ Wang and Birtcher observed the generation of spongelike porous structures on Ge at ion fluences around $10^{16}$ ions $/ \mathrm{cm}^{2} \cdot 9,10$

Nitta $e t$ al. and Kluth et al. reported the development of an anomalous cellular structure followed by the formation of a network of nanoscale rods on ion irradiated $\mathrm{GaSb}$, which is proposed to result from a defect formation mechanism based on movement of the point defects induced by ion implantation. ${ }^{11,12}$

In this article we show that for the exposure of Sb substrates to a Ga focused ion beam (FIB), the proposed defect formation mechanism, based on movement of the point defects might be active in the initial stage of FIB milling. In the later stage during FIB-induced nanowire growth we suggest a process very similar to the vapor-liquid-solid (VLS) mechanism in which various metals such as $\mathrm{Au},{ }^{13} \mathrm{Fe},{ }^{14}$ or $\mathrm{Ti}^{15}$ catalytically enhance the growth of nanowires. Thereby, a liquid alloy cluster serves as a preferential site for catalytic adsorption of reactants from the vapor phase and-when supersaturated - as the nucleation site for crystallization. The supersaturation of the eutectic melt acts as the driving force for the growth in a highly anisotropic manner. Our study differs from most of the previous reports on VLS grown nanowires in that an intense focused $\mathrm{Ga}$ ion beam initiates 
the growth of nanowires at room temperature without using any additional materials source.

\section{EXPERIMENT}

Thin lamellas of antimony samples with purity $>99.999 \%$ were prepared and cleaned by rinsing with acetone and isopropyl alcohol, followed by blow-drying with pure nitrogen. The FIB systems used were all equipped with liquid metal ion sources, either pure $\mathrm{Ga}$ or a $\mathrm{Au}_{77} \mathrm{Ge}_{14} \mathrm{Si}_{9}$ alloy with a mass separating $\boldsymbol{E} \times \boldsymbol{B}$. filter. ${ }^{16}$

The topographical and compositional evolution of the $\mathrm{Sb}$ surfaces irradiated by focused $\mathrm{Ga}, \mathrm{Au}$, or Si ion beams, respectively, is investigated by means of scanning electron microscopy (SEM), Auger electron spectroscopy (AES), conventional transmission electron microscopy (TEM), highresolution TEM (HRTEM), selected area diffraction (SAD), and energy dispersive $\mathrm{x}$-ray diffraction (EDX) measurements.

From a general point of view, the bombardment of crystalline samples by ion beams of sufficient high ion fluences and energies at first leads to a crystal-to-amorphous transformation. ${ }^{17}$ Furthermore, ion beam exposure leads to net material removal by sputtering, which is the key to all high-precision machining of micro- and nanostructures by FIB. For the purpose of patterning, the FIB is scanned over a predefined area in discrete steps with well-defined step size and dwell time, i.e., the time the beam remains on each single spot. Each scan across the selected area deposits an ion fluence which is correlated with the above-mentioned parameters. Single-pass milling denotes a scanning strategy where the desired ion fluence is deposited within one single scan. For multipass milling, the beam is scanned several times across the predefined area and the total ion fluence is dependent on the number of scan repetitions of the FIB.

\section{RESULTS AND DISCUSSION}

$\mathrm{On} \mathrm{Sb}$ surfaces exposed to an ion fluence of 6.2 $\times 10^{16}$ ions $/ \mathrm{cm}^{2}$ using a $50 \mathrm{keV}$ Ga FIB, aside from the expected material removal by physical sputtering, the formation of small dot- and fiberlike structures is observed. The dots' diameters range from 20 to $30 \mathrm{~nm}$ with an average density of approximately 200 dots per $\mu \mathrm{m}^{2}$. The diameters of the roughly $300 \mathrm{~nm}$ long fibers appear very uniform and are in the range of about $25 \mathrm{~nm}$.

Figure 1(a) shows a SEM image of the Sb surface after multipass milling of a $(2 \times 2) \mu \mathrm{m}^{2}$ wide box with a 100 times higher ion fluence of $6.2 \times 10^{18} \mathrm{Ga}$ ions $/ \mathrm{cm}^{2}$. As expected, FIB exposure leads to effective sputtering of the substrate material with a high sputter yield of approximately 14.3 sputtered $\mathrm{Sb}$ atoms per incident $\mathrm{Ga}$ ion. The rim of the several micrometers deep hole is surrounded by a dense network of nanofibers which show very uniform diameters in the range of $25 \mathrm{~nm}$. Milling the same box with the same ion fluence in single-pass mode leads to the formation of a pattern shown in Fig. 1(b). Thereby, the FIB scan starts in the upper left of the box and moves along in serpentines with a pixel and line spacing both of $10 \mathrm{~nm}$, which guarantees a nearly uniform ion fluence distribution $(>99 \%) .{ }^{18}$ The whole

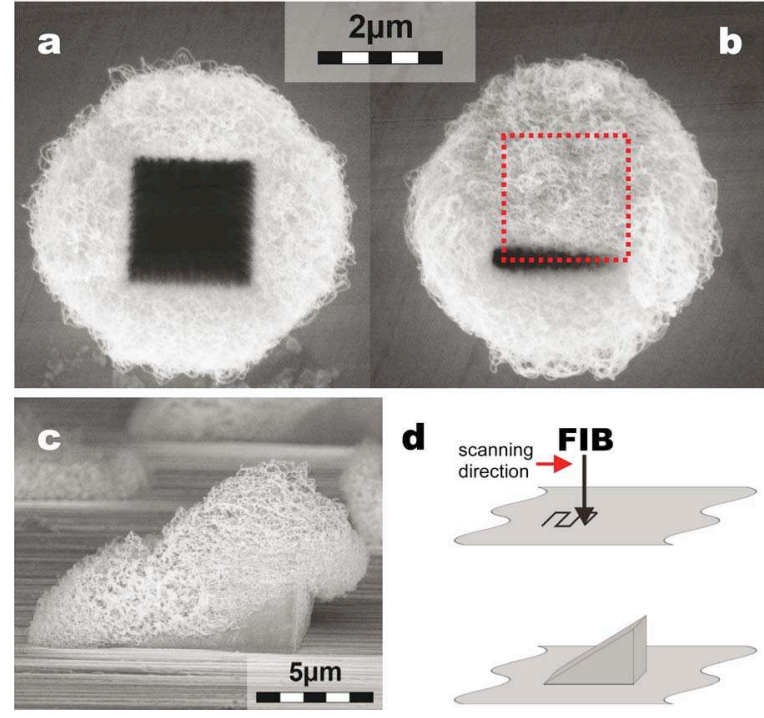

FIG. 1. (Color online) Sb surface processed using a $50 \mathrm{keV}$ Ga FIB with an ion current of $200 \mathrm{pA}$. $(2 \times 2) \mu \mathrm{m}^{2}$ milling areas irradiated by an ion fluence of $6.2 \times 10^{18}$ ions $/ \mathrm{cm}^{2}$ in (a) multipass mode and (b) single-pass mode, (c) tilted-view SEM image of a $(10 \times 10) \mu \mathrm{m}^{2}$ milling area exposed to an ion fluence of $3.1 \times 10^{18}$ ions $/ \mathrm{cm}^{2}$ processed in single-pass mode, (d) schematic sketch visualizing the FIB scanning strategy and the resulting uplifted nanofiber network.

FIB modified area is covered by nanofibers with the exception of the last line scan routed from the lower right to the lower left edge. Nanofibers reach even $2 \mu \mathrm{m}$ beyond the rim of the FIB milled area. Figure 1(c) shows the Sb surface after single-pass milling viewed under a tilt angle of $75^{\circ}$. The FIB-generated nanowire extrusions do not form a plane porous disk as one could assume from the top-view SEM image in Fig. 1(b). As shown in the schematic of Fig. 1(d), the nanofibers appear on a ramplike base normal to the plane rising along the scan direction of the FIB.

The formation of this ramplike structure is a result of the pixel-by-pixel and accordingly of the line-by-line scanning strategy. Scanning the first line of the predefined milling area leads to nanofiber growth even beyond the ion irradiated region. By the guidance of the FIB through the subsequent lines, nanofibers which were grown on the not yet exposed part in the forefront of the scanning beam are removed by sputtering. Nanofibers in already irradiated zones, i.e., behind the scanning beam, remain unaffected. These nanofibers form a network which is further densified by redeposited $\mathrm{Sb}$. Due to the ongoing FIB scanning, this nanofiber network reduces the escape angle for the sputtered $\mathrm{Sb}$ and more and more of them are picked up by the network, which leads to an upraising of the structures. Accordingly, FIB milling under oblique angles as schematically shown in Fig. 2(b) should lead to an increase or decrease of the uplifting effect due to the variation of the escape angles for sputtered $\mathrm{Sb}$. The results obtained for FIB processing of an $\mathrm{Sb}$ surface tilted by $45^{\circ}$ relative to the incident focused ion beam are shown in Fig. 2(a). Depending on the scanning direction of the FIB, the escape angles of the sputtered substrate material change. The scanning direction from higher to lower levels denoted as track (1) in the schematic of Fig. 2(b) (escape angle of $135^{\circ}$ ) results in an upraised structure. Hardly any 


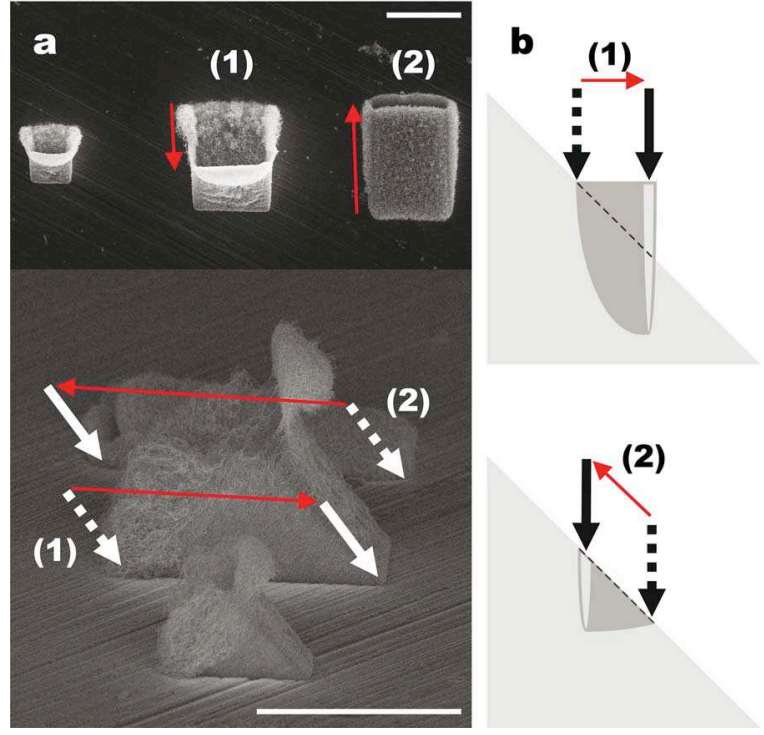

FIG. 2. (Color online) (a) SEM images of Sb surfaces milled under an angle of FIB incidence of $45^{\circ}$. The uppermost top-view SEM image and the side view show the resultant pattern achieved under different scanning directions, which are indicated by the red arrows. Track (1) denotes a FIB guidance from higher to lower surface level on the tilted sample, track (2) denotes the opposite direction. The white arrows mark the incident direction of the FIB, whereby the dotted arrows denote the start position of the scanning FIB beam. Scale bars, $10 \mu \mathrm{m}$. (b) Principle sketch to point out the guidance of the FIB and the resulting structure formation.

uplifting can be observed when scanning in the opposite direction denoted as track (2) (escape angle $45^{\circ}$ ). The smaller $(5 \times 5) \mu \mathrm{m}^{2}$ FIB milled box in Fig. 2(a) (the left box in the top view and the one in front of the side view image) shows the impact of the box length on this uplifting effect. The height of the uplifting increases with the length of the FIB milled box. For boxes with a length of $15 \mu \mathrm{m}$ or above, this height levels out at about $10 \mu \mathrm{m}$.

The cross-sectional SEM image of a $(10 \times 10) \mu \mathrm{m}^{2}$ milling box in Fig. 3 shows the porous material covering the surface and the FIB milled box. The range of the porous nanofibers network generated by the impact of the FIB reaches some microns beneath the surface level.

Extensive TEM, SAD, AES, and EDX of individual nanofibers prove that they are completely amorphous even in the nanometer scale and consist of pure antimony. The TEM

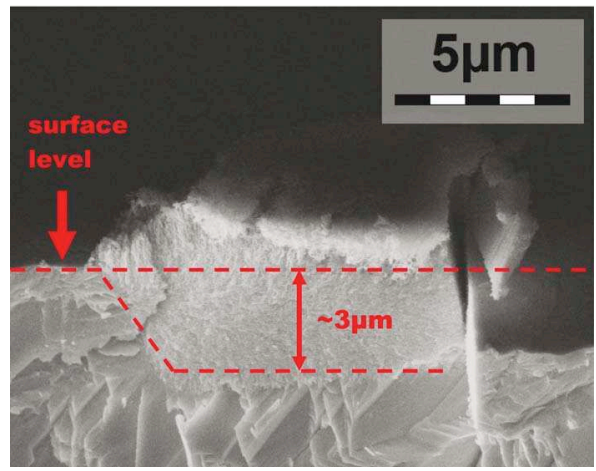

FIG. 3. (Color online) SEM image of the cross-sectional view of a FIB milled $(10 \times 10) \mu \mathrm{m}^{2}$ area accomplished by cleaving the sample. The porous network at the FIB exposed area using an ion fluence of 3.1 $\times 10^{18}$ ions $/ \mathrm{cm}^{2}$ reaches several micrometers in depth. image in Fig. 4(a) shows the exceptional uniform diameters of the nanofibers of about $25 \mathrm{~nm}$ along their entire length.

For the investigation of quantum size effects, metallic crystalline $\mathrm{Sb}$ nanowires are of emerging interest. Barati et $a l$. have reported that amorphous regions in $\mathrm{Sb}$ nanowires electrodeposited in porous anodic aluminum disks can be recrystallized by a postannealing process at temperatures of about $423 \mathrm{~K}^{19}$

The FIB modified samples covered by the nanofibers were annealed in a special furnace setup which allows processing at well-controlled temperature profiles in $\mathrm{He}$ atmosphere. Several experiments showed that the temperature ramp is a crucial parameter for the grain size of the resulting recrystallized structure, whereby the diameter and shape of the nanowires remain unaffected by the annealing. The HRTEM image in Fig. 4(b) displays an Sb nanowire after moderate thermal annealing at $453 \mathrm{~K}$ for $30 \mathrm{~min}$. The diffraction pattern in Fig. 4(c) shows the most prominent (110) and (120) reflections for $\mathrm{Sb}$ with its trigonal crystal structure, and the lattice parameter of $0.354 \mathrm{~nm}$ is consistent with the tabulated value for bulk Sb. Figure 4(d) shows the EDX spectrum of the investigated nanowire. The dashed lines mark energies for $\mathrm{Ga}$ in the EDX spectrum. As the peaks for Ga are missing there, the nanowire seems to consist of pure $\mathrm{Sb}$. The copper signal originates from the sample holder ring.

The observed nanofiber formation, even several microns aside from the FIB irradiated surface, cannot be explained by point defect as proposed by Nitta et al. ${ }^{11}$ We propose a model similar to VLS with Ga acting as catalyst. ${ }^{20}$ VLS deals with the fact that a catalytic metal particle on the sample surface-if the ambient temperature is high enough-forms a liquid alloy cluster and serves as the preferential site for adsorption of reactant from the vapor phase. It is supposed that supersaturation is the driving force for nucleation of seeds at the interface between alloy cluster and the substrate surface giving rise to a highly anisotropic growth of nanostructures.

We assume that FIB processing with the Ga beam produces mobile $\mathrm{Ga}$ species on the surface which rapidly agglomerate, forming catalytic nanoclusters. Sputtered Sb diffuses on the surface and acts as a quasivapor phase source. When the solved Sb concentration exceeds saturation, nucleation sites will be formed which initiate the precipitation of the $\mathrm{Sb}$. Nanowire growth from the base continues as long as the droplet remains in a liquid state and supersaturation is maintained. At present, we do not understand the origin of the tangling of the nanowires, although we note that extensive tangling has been observed previously in Ga-based VLS processes. ${ }^{21}$ The authors of Ref. 21 also stated that Ga droplets could simultaneously catalyze the growth of hundreds of thousands of nanowires.

To gain more information about the growth mechanism, several processes with varying substrate temperature conditions were carried out. Significant reduction of nanofiber growth was observed when the sample was cooled down to $123 \mathrm{~K}$ (Fig. 5). Lowering the ambient temperature seems to counteract the FIB-induced local heating. Thus, the applied thermal energy needed for the catalytic growth process may be too low for the formation of a dense nanofiber network. 

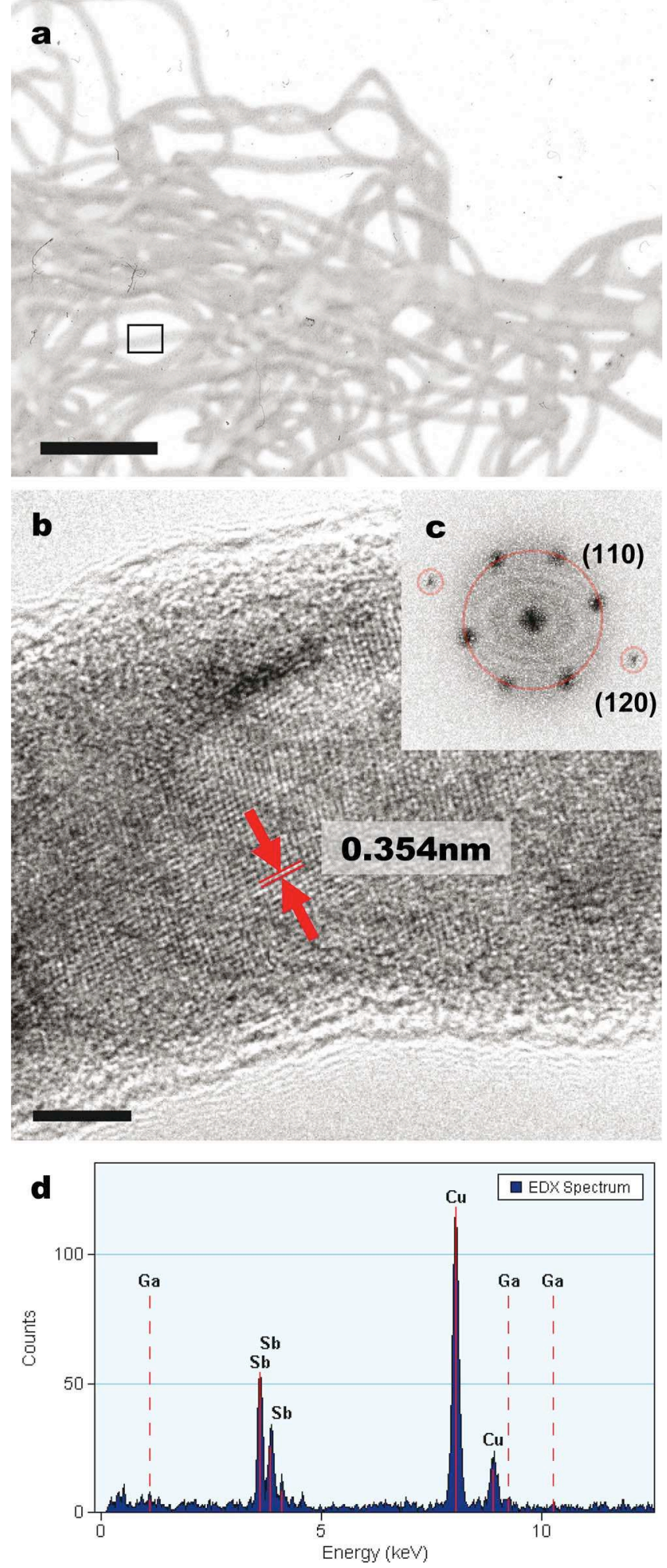

FIG. 4. (Color online) The low magnified TEM image (a) gives an overview of the nanofibers network. The scale bar corresponds to $200 \mathrm{~nm}$. The HRTEM micrograph in (b) shows the part of an Sb nanofiber [marked by the rectangle in (a)] after moderate thermal annealing at $453 \mathrm{~K}$. The lattice planes as highlighted in the HRTEM image show a distance of $0.354 \mathrm{~nm}$ which corresponds to $\mathrm{Sb}$ (110) direction. The scale bar denotes $5 \mathrm{~nm}$. The diffraction pattern (c) clearly shows the single-crystalline nature $[(110)$ and (120) reflections] of the nanowires after the postgrowth annealing at $453 \mathrm{~K}$ for $30 \mathrm{~min}$ in He atmosphere. The EDX spectrum (d) shows that the nanowire consists of pure $\mathrm{Sb}$; the copper signal is an artifact from the TEM sample holder.

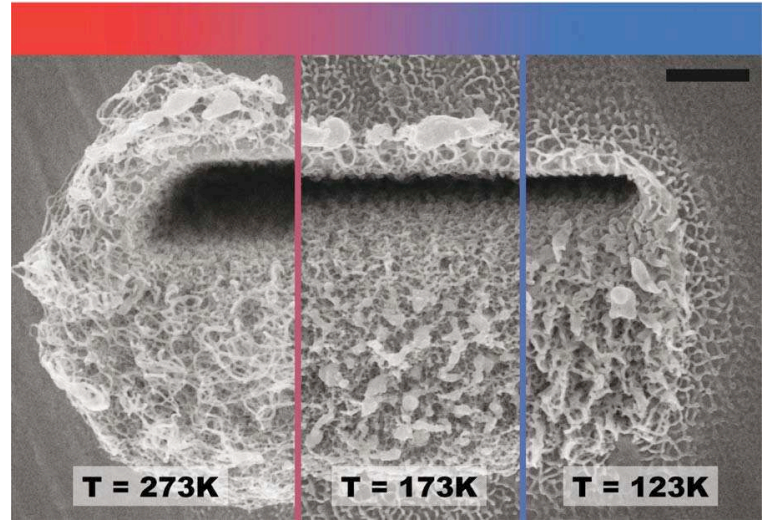

FIG. 5. (Color online) SEM images of Sb exposed to the Ga FIB at 273, 173 , and $123 \mathrm{~K}$. The FIB processing of samples cooled by liquid nitrogen leads to reduction of the density of the nanofiber network. Scale bar, 500 nm.

Consequently, the Ga provided by the FIB agglomerates to dropletlike structures without any catalytic synthesis of nanofibers.

Additional experiments were carried out with a reduced acceleration voltage of the $\mathrm{Ga}$ ions down to $10 \mathrm{kV}$. As expected, the formation of nanofibers diminished and the surface was removed by sputtering.

Alternative liquid metal ion sources were used to prove the catalytic activity of the Ga species. For the Sb samples exposed to $\mathrm{Au}$ and $\mathrm{Si}$ ions at $60 \mathrm{kV}$ acceleration voltage, no nanofiber formation was observed. This can be explained by the fact that under the given experimental conditions $\mathrm{Au}$ and Si might not form liquid droplets due to their high melting point.

\section{SUMMARY}

In summary, the FIB irradiation of Sb with $50 \mathrm{keV} \mathrm{Ga}$ ions at room temperature leads to the formation of a porous network of pure $\mathrm{Sb}$ nanofibers. The as-grown nanofibers are amorphous with remarkable uniform diameters in the range of about $25 \mathrm{~nm}$ along their entire length. The resulting porous network is uplifted several microns above the sample surface. In accordance with the catalytic VLS approach, we suppose that the formation of $\mathrm{Sb}$ nanowires necessitates a catalytic particle, mostly a eutectic alloy, with a low melting point. Recrystallization of the $\mathrm{Sb}$ nanofibers could be achieved by moderate thermal annealing at temperatures of about $473 \mathrm{~K}$. Depending on the temperature ramp and heating duration, finely grained crystallites as well as singlecrystalline regions along the nanowires can be obtained.

\section{ACKNOWLEDGMENTS}

This work is partly funded by the Austrian Science Fund (Project No. 18080-N07) and the Sixth EU Framework Program for Research and Technological Development (FP6) project "Charged Particle Nanotech" (CHARPAN). The authors thank the Center for Micro- and Nanostructures (ZMNS) for providing the clean-room facilities and the Austrian Society for Micro- and Nanoelectronics (GMe) for financial support. 
${ }^{1}$ D. Bouchet, E. Roy, K. Yu-Zhang, and Y. Leprince-Wang, Eur. Phys. J. Appl. Phys. 30, 193 (2005).

${ }^{2}$ Y. Zhang, L. Li, G. H. Li, and L. D. Zhang, Phys. Rev. B 73, 113403 (2006).

${ }^{3}$ J. H. Xu and C. S. Ting, Appl. Phys. Lett. 63, 129 (1993).

${ }^{4}$ Z. X. Jiang and P. F. A. Alkemade, Appl. Phys. Lett. 73, 315 (1998).

${ }^{5}$ J. Erlebacher, M. J. Aziz, E. Chason, M. B. Sinclair, and J. A. Floro, Phys. Rev. Lett. 82, 2330 (1999).

${ }^{6}$ S. Rusponi, C. Boragno, and U. Valbusa, Phys. Rev. Lett. 78, 2795 (1997).

${ }^{7}$ S. Rusponi, G. Constantini, C. Boragno, and U. Valbusa, Phys. Rev. Lett. 81, 2735 (1998).

${ }^{8}$ B. R. Appleton, O. W. Holland, D. B. Poker, J. Narayan, and D. Fathy, Nucl. Instrum. Methods Phys. Res. B 7-8, 639 (1985).

${ }^{9}$ L. M. Wang and R. C. Birtcher, Appl. Phys. Lett. 55, 2494 (1989)

${ }^{10}$ L. M. Wang and R. C. Birtcher, Philos. Mag. A 64, 1209 (1991).

${ }^{11}$ N. Nitta, M. Taniwaki, Y. Hayashi, and T. Yoshiie, J. Appl. Phys. 92, 1799 (2002).
${ }^{12}$ S. M. Kluth, J. D. Fitz Gerald, and M. C. Ridgway, Appl. Phys. Lett. 86, 131920 (2005).

${ }^{13}$ A. I. Persson, M. L. Larsson, S. Stenström, B. J. Ohlsson, L. Samuelson, and L. R. Wallenberg, Nat. Mater. 3, 677 (2004).

${ }^{14}$ A. M. Morales and C. M. Lieber, Science 279, 208 (1998).

${ }^{15}$ T. I. Kamins, R. S. Williams, Y. Cheng, Y. L. Chang, and Y. A. Chang, Appl. Phys. Lett. 76, 562 (2000).

${ }^{16}$ L. Bischoff, Ultramicroscopy 103, 59 (2005).

${ }^{17}$ J. S. Williams, MRS Bull. 17, 47 (1992).

${ }^{18}$ S. Lipp, L. Frey, C. Lehrer, B. Frank, E. Demm, and H. Ryssel, J. Vac. Sci. Technol. B 14, 3996 (1996)

${ }^{19}$ M. Barati, J. C. L. Chow, P. K. Ummat, and W. R. Datars, J. Phys.: Condens. Matter 13, 2955 (2001)

${ }^{20}$ M. K. Sunkara, S. Sharma, R. Miranda, G. Lian, and E. C. Dickey, Appl. Phys. Lett. 79, 1546 (2001)

${ }^{21}$ Z. W. Pan, Z. R. Dai, C. Ma, and Z. L. Wang, J. Am. Chem. Soc. 124, 1817 (2002) 